\title{
A MECHANISM FOR SYMMETRY BREAKING IN ANTIFERROMAGNETIC HEISENBERG SYSTEMS
}

\author{
P.L. ISKE and W.J. CASPERS \\ Center for Theoretical Physics, University of Twente, Enschede, The Netherlands
}

Received 4 August 1986

\begin{abstract}
An approximation method, based on dimer coverings, for the ground state of the antiferromagnetic Heisenberg lattice is described. The working of the method is demonstrated by some small-system calculations. The method introduces a possible mechanism for symmetry breaking for two- and higher-dimensional systems.
\end{abstract}

\section{Introduction}

Much attention has been paid in the literature to the ground-state properties of antiferromagnetic Heisenberg systems $\left(s=\frac{1}{2}\right)$.

An important subject to investigate is whether long-range order exists in the ground state or not. The classical case is quite simple: The ground state is always a Néel state. If it is possible to subdivide the lattice into two sublattices, $\alpha$ and $\beta$, such that each point of sublattice $\alpha$ is surrounded by points of $\beta$, and vice versa, one can define a Néel state to be a state with a maximum, oppositely directed, sublattice magnetization. See fig. 1 .

The quantum-mechanical problem is much more complicated. Even at zero temperature quantum fluctuations allow the system to 'jump out' of the Néel state and so long-range order can be broken down.

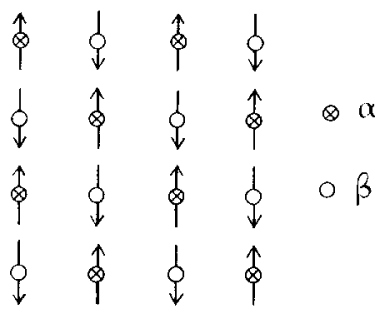

Fig. 1. A Néel state in the square lattice.

0378-4371/87/\$03.50 (C) Elsevier Science Publishers B.V.

(North-Holland Physics Publishing Division) 
Exact results are scarce in this field of statistical physics. The first exact result in this area was the calculation of the ground-state wave function of the linear chain with nearest-neighbour interactions only, done by Bethe in $1931^{1}$ ). From Bethe's solution it became clear that there exists no long-range order in one-dimensional antiferromagnetic systems and this would be confirmed by other calculations, e.g. done by Lieb, Schultz and Mattis ${ }^{2}$ ).

Mermin and Wagner proved the strong theorem that says that no long-range order can exist at finite temperatures for one and two-dimensional systems ${ }^{3}$ ). Peierls and Marshall proved the ground state of an antiferromagnetic Heisenberg system to be a singlet ${ }^{4}$ ).

The situation for three-dimensional systems still is very unclear, almost nothing is known exactly about ground-state properties of e.g. the Heisenberg model for a simple cubic lattice. Besides the one-dimensional antiferromagnet the, almost trivial, spherical model for antiferromagnets can be treated exactly. The ground state of this model, where every $\beta$-lattice site is a neighbour of every $\alpha$-site and vice versa, shows a classical, Néel ordering.

The question arises for which dimensions the ground state possesses longrange order. This order may exist for two-dimensional systems, but according to Mermin and Wagner $^{3}$ ), it will vanish for any finite temperature. By the absence of an exact expression for the ground state in two dimensions, one is forced to make use of approximation methods. Thus far various types of approximations have been made to estimate the ground-state energy. Marshall $^{4}$ ), Davis ${ }^{5}$ ), Bullock ${ }^{6}$ ), Kubo ${ }^{7,8}$ ), Bartkowski ${ }^{9}$ ), Oitmaa and Betts ${ }^{10}$ ), Floria and Navarro ${ }^{11}$ ): All these authors were able to give a good estimate of the ground-state energy but none of them could give much detail of the ground-state wave function.

To understand symmetry breaking and its relation with long-range order, however, one needs a good impression of the structure of the ground-state wave function. Only Van den Broek ${ }^{12}$ ) used a method that not only gives a good estimate of the energy but also some information about the wave function, such as correlation functions.

In antiferromagnetic quantum systems we can discern two tendencies: In the first place the system tries to realize a maximum sublattice magnetization, secondly it strives to form neighbour pairs in the singlet state.

By using a method, already introduced by Hite et al. ${ }^{13}$ ) in the theory of $\pi$-networks, we try to show long-range order can be built up by taking a suitable linear combination of so-called singlet-pair states, where every spin forms a singlet with one of its neighbours. If we take such a linear combination we are certain that the total state will be a singlet, in accordance with the theorem of Peierls and Marshall.

We shall demonstrate the principle by showing the results of calculations for 
some small systems. Of course we will test whether our ground-state energy will be in agreement with already existing approximations.

Another point of interest forms the Marshall criterion ${ }^{4}$ ). This criterion predicts the signs of the configuration coefficients in the linear combination which forms the ground state.

If we want to construct a reliable approximated ground state, at least we have to take care that there is a great overlap between the approximated and the exact ground state, so it is necessary that the coefficients in our ground state obey the Marshall criterion in a huge majority.

\section{Description of the approximation}

We consider the following Hamiltonian:

$$
H=J \sum_{\langle i j\rangle} s_{i} \cdot s_{j},
$$

where the sum runs over all neighbour pairs. For convenience we will further take $J=1$.

We approximate the ground state by a linear combination of states in which every spin forms a singlet with one of its nearest neighbours.

Such a singlet pair can be regarded as a dimer and every state in the linear combination corresponds with a dimer covering of the lattice. The lattice contains $2 N$ lattice sites ( $N$ in each sublattice) so there are $N$ dimers. If we denote a dimer covering with the symbol $|n\rangle$, we may write the ground state in our approximation as

$$
\left|\psi_{0}\right\rangle=\sum_{n} c_{n}|n\rangle
$$

We number the spins $1,2, \ldots, 2 N$, so that an even index corresponds with a spin in the $\beta$-sublattice and an odd index with an $\alpha$-spin.

The state $\left|\psi_{0}\right\rangle$ may be written as a linear combination of eigenstates of the individual $s_{i}^{z}$. We call these states Ising configurations throughout this work and we represent them by kets like $|++-+--\ldots\rangle$, where the sign \pm on the $i$ th place stands for an eigenvalue $\pm \frac{1}{2} \hbar$ of $s_{i}^{z}$. These kets are orthonormal.

The total spin $S$ and its $z$-component $M$ are good quantum numbers. Because the ground state is a singlet, we restrict ourselves to states with $M=0$, i.e. to Ising configurations with as many spins "up" $(+)$ as "down" $(-)$. Each dimer covering $|n\rangle$ contains $N$ dimers and therefore $|n\rangle$ can be written as a linear combination of $2^{N}$ Ising configurations. The coefficient of each Ising configuration in $|n\rangle$ is +1 or -1 , apart from an overall normalization factor.

We choose the signs so that in each dimer covering the Néel configuration 

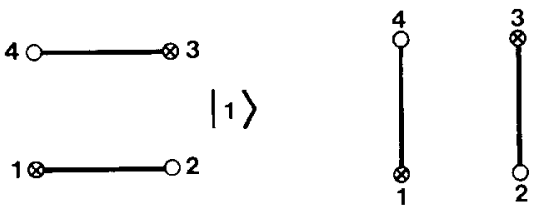

$|2\rangle$

Fig. 2. The two dimer coverings of the $2 \times 2$-square.

$|+-+-+-\ldots\rangle$ and all the configurations one can get by an even number of neighbour interchanges, are given the coefficient +1 . All the other Ising configurations belonging to the dimer covering become coefficient -1 .

Within one dimer covering the Marshall criterion is by this construction automatically fulfilled. We give a simple example, the $2 \times 2$-lattice, see fig. 2 , which allows for two dimer coverings, $|1\rangle$ and $|2\rangle$.

$$
\begin{aligned}
& |1\rangle=\frac{1}{2}\{|+-+-\rangle-|+--+\rangle-|-++-\rangle+|-+-+\rangle\}, \\
& |2\rangle=\frac{1}{2}\{|+-+-\rangle-|++--\rangle-|--++\rangle+|-+-+\rangle\} .
\end{aligned}
$$

For reasons of symmetry we can divide the dimer coverings into classes. Within a class each dimer covering has the same coefficient $\tilde{c}_{n}$ (see (2)).

In the case of the $2 \times 2$-lattice it is obvious that the two dimer coverings are equivalent for reasons of symmetry, so the ground state may be written as

$$
\begin{aligned}
\left|\psi_{0}\right\rangle= & \frac{\tilde{c}}{2}\{2(|+-+-\rangle+|-+-+\rangle)-(|+--+\rangle+|-++-\rangle+|++--\rangle \\
& +|--++\rangle)\} .
\end{aligned}
$$

This state turns out to be the exact ground state and is normalized for $\tilde{c}=3^{-1 / 2}$.

We now already notice the special role the Néel configurations play: They are the only configurations that can be covered by all dimer coverings, so if the number of dimer coverings increases, the relative amplitude of the Néel configurations in the ground state increases too.

We now derive an expression for the expectation value of the energy in the approximated ground state.

Therefore we rewrite (2) as

$$
\left|\psi_{0}\right\rangle=\sum_{i} \varepsilon_{i}\left(\sum_{n} c_{n} \xi_{i, n}\right)\left|\phi_{i}\right\rangle
$$

In (5) the first summation runs over all Ising configurations $\left|\phi_{i}\right\rangle$, i.e. distributions of an equal number of + and - signs over the lattice sites, the second sum runs over the dimer coverings. We define: 
$\xi_{i, n}=1$ if the Ising configuration $i$ is covered by $|n\rangle$;

$\xi_{i, n}=0$ if the Ising configuration $i$ is not covered by $|n\rangle$.

In (5), $\varepsilon_{i}$ is the "Marshall-sign", $\varepsilon_{i}=(-1)^{p_{i}}$, where $p_{i}$ is the number of interchanges of neighbours that is necessary to reach the Ising configuration $i$ starting from the Néel configuration $|+-+-+-\ldots\rangle$.

If we define

$$
a_{i}=\varepsilon_{i} \sum_{n} c_{n} \xi_{i, n}
$$

then (5) simplifies to

$$
\left|\psi_{0}\right\rangle=\sum_{i} a_{i}\left|\phi_{i}\right\rangle
$$

If we regard only the $z-z$-component of the Hamiltonian (1), then the energy of an Ising configuration is given by

$$
H^{z z}\left|\phi_{i}\right\rangle=E_{i}^{z z}\left|\phi_{i}\right\rangle, \quad E_{i}^{z z}=\sum_{\langle j k\rangle} s_{j, i}^{z} s_{k, i}^{z}
$$

where $s_{j, i}^{z}$ is the eigenvalue of the one-particle operator $s_{j}^{z}$ for the state $\left|\phi_{i}\right\rangle$.

The expectation value of the $z-z$-component of the ground-state energy can now be written as

$$
E_{0}^{z z}=\frac{\left\langle\psi_{0}\left|H^{z z}\right| \psi_{0}\right\rangle}{\left\langle\psi_{0} \mid \psi_{0}\right\rangle}=\frac{\sum_{i} E_{i}^{z z} a_{i}^{2}}{\sum_{i} a_{i}^{2}}
$$

For the expectation value of the total ground-state energy we can, on the ground of rotational symmetry, easily write

$$
E_{0}=3 E_{0}^{z z}
$$

Now we derive an expression for the probability that the system is in a Néel state. In a Néel state there is a maximum sublattice magnetization which is $\frac{1}{2} N$.

The magnetization of the two sublattices are antiparallel in a Néel state. In quantummechanical language: $S_{\alpha}^{2}$ and $S_{\beta}^{2}$ have a maximum eigenvalue: $\frac{1}{2} N\left(\frac{1}{2} N+1\right) \hbar^{2}$, whereas the eigenvalue of $S_{\alpha}^{z}$ and $S_{\beta}^{z}$ obey: $M_{\alpha}=-M_{\beta}$. There are $N+1$ of these substates with anti-parallel sublattice magnetizations, written as 


$$
\left|\frac{1}{2} N M\right\rangle \otimes\left|\frac{1}{2} N-M\right\rangle \quad\left(M=-\frac{1}{2} N,-\frac{1}{2} N+1, \ldots, \frac{1}{2} N\right) .
$$

From the Clebsch-Gordan transformation

$$
\begin{aligned}
\left|j_{1} j_{2} j m\right\rangle & =\left|\frac{1}{2} N \frac{1}{2} N 00\right\rangle \\
& =\sum_{M}\left\langle\frac{1}{2} N M \frac{1}{2} N-M \mid \frac{1}{2} N \frac{1}{2} N 00\right\rangle\left|\frac{1}{2} N M\right\rangle \otimes\left|\frac{1}{2} N-M\right\rangle,
\end{aligned}
$$

with (cf. ref. 14)

$$
\left\langle\frac{1}{2} N M \frac{1}{2} N-M \mid \frac{1}{2} N \frac{1}{2} N 00\right\rangle=(-)^{N / 2-M}(N+1)^{-1 / 2},
$$

we conclude that all these Néel states have the same probability (this can also be concluded on grounds of rotational symmetry). The total probability for a Néel state is therefore given by

$$
P_{\text {Néel }}=\frac{(N+1) a_{\text {Néel }}^{2}}{\sum_{i} a_{i}^{2}},
$$

where $a_{\text {Néel }}$ is the amplitude (6) of one of the Néel configurations.

We now discuss the relation between long-range order and the Néel probability.

First we consider a state (2), made up of only one dimer covering $|\phi\rangle$. This (normalized) state can be written as a direct product of singlet states for pairs $j$,

$$
|\phi\rangle=\prod_{j=1}^{N}|0\rangle_{j}
$$

with

$$
|0\rangle_{j}=\frac{1}{\sqrt{2}}\{|+-\rangle-|-+\rangle\}_{j} .
$$

In this state each spin on a given sublattice has an equal probability $\frac{1}{2}$ to be "up" as to be "down". There is no correlation between spins of different pairs; in that sense the state is completely disordered.

The amplitude of e.g. the first Néel state,

$$
\left|N_{1}\right\rangle=|+-\rangle_{1} \otimes|+-\rangle_{2} \otimes \cdots \otimes|+-\rangle_{N},
$$

can be easily calculated by taking the inner product with $|\phi\rangle$, 


$$
a_{\text {Née }_{1}}=\left\langle N_{1} \mid \phi\right\rangle=\left(\frac{1}{\sqrt{2}}\right)^{N},
$$

so the Néel probability $P_{\text {Néel }}$ in the disordered case is

$$
P_{\mathrm{Nécl}}^{\mathrm{d}}=(N+1) \frac{1}{2}^{N} \quad(\mathrm{~d}=\text { disordered }) \text {. }
$$

We now define the "Néel probability per dimer" in the thermodynamic limit as

$$
p_{\text {Néel }}^{*}=\lim _{N \rightarrow \infty} N^{N} P_{\text {Néel }}
$$

From (19) we see that in the "disordered" case $p_{\text {Néel }}^{*}=\frac{1}{2}$. As a sufficient condition for long-range order in our approximation we now consider

$$
p_{\text {Néel }}^{*}>\frac{1}{2}
$$

In our view (21) corresponds to the condition of broken symmetry, i.e. a wave packet of only one of the Néel states and a group of neighbouring states with a relatively small amplitude for large $N$, is asymptotically stable. It would take an infinitely long time for the systems to pass into another Néel state. For a more detailed discussion of symmetry breaking we refer to Anderson ${ }^{15}$ ).

\section{Dimension dependence}

We already noticed that no long-range order exists in the ground state of the one-dimensional antiferromagnetic Heisenberg model.

Qualitatively we can explain the absence of SB in the linear chain by using our approximation and by realizing that the linear chain has only two dimer coverings (we suppose periodic boundary conditions). See fig. 3. The Néel amplitude is only 2 and therefore too small to cause SB. We can calculate the Néel probability exactly in this approximation: Both dimer coverings contain $2^{N}$ Ising-configurations. The only common configurations are the Néel configurations. Both Néel configurations have amplitude with absolute value $2 c$ ( $c$ is a normalization constant. Notice that $|1\rangle$ and $|2\rangle$ are equivalent for reasons of

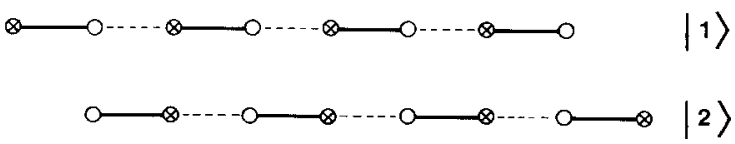

Fig. 3. The two dimer coverings of the linear chain with periodic boundary conditions. 
symmetry). The other $2 \cdot 2^{N}-4$ Ising configurations that can be covered have an amplitude $\left|a_{i}\right|=1 c$. Substitution in (14) gives

$$
P_{\text {Néel }}=\frac{(N+1) \cdot 2^{2}}{\left(2 \cdot 2^{N}-4\right) \cdot 1^{2}+2 \cdot 2^{2}}=\frac{4 N+4}{2^{N+1}+4} .
$$

From (20) it then follows that

$$
p_{\text {Néel }}^{*}=\frac{1}{2} \text {. }
$$

The expectation value for the ground-state energy can be calculated easily in this case. In the thermodynamic limit the dimer coverings $|1\rangle$ and $|2\rangle$ become orthonormal, so the energy per lattice site may be determined from the energy for one covering.

In a covering every spin has two neighbours: One of them is part of the same dimer and has therefore the opposite sign which gives a contribution -0.25 to the energy, that is -0.125 per spin. The other neighbour takes part in another dimer and may therefore have the same as well as the opposite sign, so this bond does not contribute to the energy. So we find for the energy per site

$$
\varepsilon^{z z}=-0.125 \rightarrow \varepsilon=-0.375 .
$$

If we compare this value with the exact value, calculated by Hulthén ${ }^{16}$ ),

$$
\varepsilon=\frac{1}{4}-\ln 2 \approx-0.443,
$$

then we see that our value (24) is about $15 \%$ too high. In this case we can obtain a better approximation by introducing non-neighbour pairs (with one spin on the $\alpha$-sublattice and the other on $\beta$ ) in the singlet state (cf. section 4), so then there will be more coverings. The variational space of (2) then becomes larger and we may expect that the minimum energy in this space is lower and therefore a better approximation of the exact ground-state energy. This effect, due to the overlap between the dimer coverings is usually called resonance (Hite et al. ${ }^{13}$ ). Although in this case the Néel amplitude will be larger too, we don't expect the number of coverings will be so large that (21) will hold.

In two and more dimensions the situation will be quite different, because the co-ordination number increases with increasing dimension, and this results in a rapid increase of the number of dimer coverings per lattice site.

In two dimensions, for example, we have the famous Kasteleyn/Fisher ${ }^{17,18}$ ) result 


$$
Z \approx(1.79)^{N}, \quad N \rightarrow \infty
$$

where $Z$ is the number of dimer coverings.

By taking a linear combination of all these coverings we expect to be able to construct a good approximation of the ground state.

The limit $d \rightarrow \infty$ ( $d=$ dimension) can be represented by a lattice in which all sites of a given sublattice have all the sites of the other sublattice as neighbours. In this so-called spherical limit our method generates the exact ground state.

As a result of the increasing number of dimer coverings it may be possible that the relative weights of the Néel states will be large enough to cause SB, and this may occur, starting with a critical dimension $d_{c}=2$. The (exact) ground state of the spherical model is certainly ordered, because here we have $p_{\text {Néel }}^{*}=1$.

Anyhow, we have a mechanism that can explain for the occurrence of SB, starting from the tendency in the system to form singlet pairs: It is caused by the fact that some Ising configurations (the Néel configurations) can be covered by dimers more than others. So, in fact, it is a combinatorial effect.

\section{Examples: some small-system calculations}

To illustrate the working of our approximations for $d \geqq 2$, we have done some computer calculations for the $4 \times 4$-square and for the $2 \times 2 \times 2$-cube. If we suppose periodic boundary conditions, the $4 \times 4$-square admits 272 dimer coverings. These coverings can be subdivided into 13 classes with weights $\tilde{c}_{1}$, $\tilde{c}_{2}, \ldots, \tilde{c}_{13}$. Coverings within a class are related by the symmetry of the system. In the most simple approximation, in which we give all classes the same weight,

$$
\mathrm{A}: \quad \tilde{c}_{1}=\bar{c}_{2}=\cdots=\bar{c}_{13},
$$

we automatically are in agreement with the Marshall criterion. In table I, first row, we give the results for his approximation.

Secondly we may allow for different weights, so we get a variational problem if we minimize the energy (9).

This energy now has the form

$$
E^{z z}=\frac{\boldsymbol{x}^{\mathrm{T}} \mathbf{A} \boldsymbol{x}}{\boldsymbol{x}^{\mathrm{T}} \mathbf{B} \boldsymbol{x}}, \quad \text { with } \boldsymbol{x}=\left(\tilde{c}_{1}, \tilde{c}_{2}, \ldots, \tilde{c}_{13}\right),
$$

where $\mathbf{A}$ is a real, symmetric $(13 \times 13)$-matrix and $\mathbf{B}$ is a real, symmetric 
TABLE I

Results for the $4 \times 4$-square with periodic boundary conditions.

\begin{tabular}{lllllll}
\hline Approximation & & $\varepsilon$ & $\gamma_{1}$ & $\gamma_{2}$ & $p_{\text {Neel }}^{*}$ & Marshall \\
\hline dimers only & A: $\tilde{c}_{1}=\tilde{c}_{2}=\cdots=\tilde{c}_{13}$ & -0.6686 & -0.3343 & +0.1488 & 0.899 & $100 \%$ \\
& B: $\left(\tilde{c}_{1} \tilde{c}_{2}, \ldots, \tilde{c}_{13}\right)_{\min }$ & -0.6767 & -0.3383 & +0.1645 & 0.903 & $99.91 \%$ \\
dimers only & C: $\tilde{c}_{1}=\tilde{c}_{2}$ & -0.6964 & -0.3482 & $*$ & 0.945 & $100 \%$ \\
$\begin{array}{l}\text { (index 1) and } \\
\text { dimers + one }\end{array}$ & D: $\tilde{c}_{1}=1 ; \tilde{c}_{2}=x_{\min }$ & -0.6965 & -0.3482 & $*$ & 0.951 & $100 \%$ \\
knight's move & & & & & & \\
(index 2) & & & & & & \\
\hline
\end{tabular}

positive definite $(13 \times 13)$-matrix. The structure of the matrices $\mathbf{A}$ and $\mathbf{B}$ makes the minimum problem for $E^{z z}$ equivalent to solving the following generalized eigenvalue problem:

$$
\mathbf{A} \boldsymbol{x}=E_{0}^{z z} \mathbf{B} \boldsymbol{x},
$$

where $E_{0}^{z z}$ is the minimum eigenvalue. It is possible that one or more of the $\bar{c}_{i}$ have a negative sign in the solution of (29), and possibly the Marshall condition is not fulfilled. Indeed in the $4 \times 4$-system some of the $\tilde{c}_{i}$ have an opposite sign (perhaps a finite system effect) but fortunately only eight out of 8918 Ising configurations that can be covered by dimers, turn out to have the wrong sign in the total linear combination (5), i.e. only $0.09 \%$. Furthermore, the relative Néel amplitude is still very large.

The results of this calculation (B) are given in the second row of table I. Finally we have investigated the influence of allowing one pair of spins at distance $\sqrt{5}$ (the knight's move), to form a singlet. The two spins in such a pair belong to the different sublattices. Also for this case we did two calculations: In the first (C) we gave all 1808 coverings the same weight, in the second (D) we discriminated between the 272 real dimer coverings, with relative weight 1 , and the 1536 coverings with one knight's move, with relative weight $x$. We found a minimum in the energy for $x \approx 1.45$. We find that the energy does not change very much by introducing the knight's moves.

In table I, $\varepsilon$ is the energy per site, $\gamma_{1}$ and $\gamma_{2}$ are the nearest- and next-nearest neighbour correlation functions respectively. In the last column 'we have written down the fraction of Ising configurations with the right (Marshall-) sign. As one can see there is not much discrepancy between the first approximation (A) and the others. This may be an indication that it is sufficient to consider only dimers and to give all coverings the same weight.

If we use the spin-wave approach to get an estimate for the ground-state energy (cf. ref. 12, ch. IV), we end up with an expression that in the case of 
the $4 \times 4$-square may be written as

$$
\begin{gathered}
\varepsilon=-1.5+\frac{1}{32} \sum_{n_{x}, n_{y}} \sqrt{ }\left\{4-\left(\cos \frac{\pi}{2} \cdot n_{x}+\cos \frac{\pi}{2} \cdot n_{y}\right)^{2}\right\} \\
\left(n_{x}, n_{y}=0,1,2,3\right) .
\end{gathered}
$$

If we work out the summation we find $\varepsilon=-0.6920$, which is very well in agreement with the results of table $I$.

In table II we have given the results for the $4 \times 4$-square lattice with free ends.

Now there are 36 coverings, split up into 10 classes (cf. E and F). Again we have considered the influence of adding one knight's move. There are 144 coverings of this type, to be divided in 18 classes, corresponding with a variational problem with 28 variables $(\mathrm{G}$ and $\mathrm{H})$.

Finally, we considered the effect of allowing the number of knight's moves to be any number $i, i=0,1,2, \ldots, 8$. All coverings with $i$ moves we gave the same weight $\tilde{c}_{i}$ (approximations $I$ and $J$ ). Here again we may conclude that the first approximation (E) differs not much from the others, with the exception of the non-realistic approximation (I).

In table III we compare the results of approximations (A) and (E) with results obtained by other authors for the thermodynamic limit. These examples represent most straightforwardly our idea.

We see that $(A)$ is in good agreement with these values (though the system contains only $4 \times 4=16$ sites!), while (E) predicts an energy that is somewhat too high; we could interpret this as a boundary effect. Together with Van den Broek's results, our approximation is the only one that gives a reasonable value for the spin-correlation functions. Because in the square lattice the coordination number is four, it is easily seen that we should have: $\gamma_{1}=\frac{1}{2} \varepsilon$ in this lattice.

\section{TABLE II}

Results for the $4 \times 4$-square with free ends.

\begin{tabular}{lllllll}
\hline Approximation & & $\varepsilon$ & $\gamma_{1}$ & $\gamma_{2}$ & $p_{\text {Neel }}^{*}$ & Marshall \\
\hline dimers only & E: $\bar{c}_{1}=\bar{c}_{2}=\cdots=\tilde{c}_{10}$ & -0.5437 & -0.3625 & +0.1522 & 0.813 & $100 \%$ \\
& F: $\left(\bar{c}_{1}, \bar{c}_{2}, \ldots, \bar{c}_{10}\right)_{\min }$ & -0.5459 & -0.3639 & +0.1522 & 0.820 & $100 \%$ \\
dimers only and & G: $\tilde{c}_{1}=\tilde{c}_{2}=\cdots=\tilde{c}_{28}$ & -0.5621 & -0.3747 & +0.1872 & 0.872 & $100 \%$ \\
dimers + one & H: $\left(\bar{c}_{1}, \bar{c}_{2}, \ldots, \tilde{c}_{28}\right)_{\min }$ & -0.5664 & -0.3775 & +0.1916 & 0.876 & $100 \%$ \\
knight's move & & & & & & \\
$0,1,2, \ldots, 8$ & $\mathrm{I}: \bar{c}_{0}=\bar{c}_{1}=\ldots=\bar{c}_{8}$ & -0.4840 & -0.3226 & $*$ & 0.949 & $100 \%$ \\
knight's moves & $\mathrm{J}:\left(\tilde{c}_{0}, \bar{c}_{1}, \ldots, \tilde{c}_{8}\right)_{\min }$ & -0.5661 & -0.3774 & $*$ & $*$ & $100 \%$ \\
\hline
\end{tabular}


TABLE III

Estimates for ground-state properties of the square lattice.

\begin{tabular}{lllll}
\hline Source & & $\varepsilon$ & $\gamma_{1}$ & $\gamma_{2}$ \\
\hline Perturbational & Davis $^{5}$ ) & -0.664 & -0.51 & $*$ \\
& Bullock $^{6}$ ) & -0.666 & -0.50 & $*$ \\
Spin-wave & Van den Broek $^{12}$ ) & -0.651 & -0.33 & +0.15 \\
& Kubo $^{7}$ ) & -0.671 & $*$ & $*$ \\
& Van den Broek $^{12}$ ) & -0.658 & -0.33 & +0.20 \\
Variational & ${\text { Floria } / \text { Navarro }^{11} \text { ) }}$ & -0.635 & $*$ & $*$ \\
& Kubo $^{8}$ ) & -0.647 & $*$ & $*$ \\
& Marshall $^{4}$ ) & -0.656 & $*$ & $*$ \\
Extrapolation & Bartkowski $\left.^{9}\right)$ & -0.659 & $*$ & $*$ \\
This work & Oitmaa $/$ Betts $^{10}$ ) & -0.655 & $*$ & $*$ \\
& $(\mathrm{~A})$ & -0.669 & -0.334 & +0.149 \\
& (E) & -0.544 & -0.362 & +0.152 \\
\hline
\end{tabular}

TABLE IV

Results for the $2 \times 2 \times 2$-cube.

\begin{tabular}{lllll}
\hline Approximation & $\varepsilon$ & $\gamma_{1}$ & $p_{\text {Néel }}^{*}$ & Marshall \\
\hline $\bar{c}_{1}=\tilde{c}_{2}$ & -0.590 & -0.393 & 0.941 & $100 \%$ \\
$\left(\tilde{c}_{1}, \tilde{c}_{2}\right)_{\min }$ & -0.593 & -0.395 & 0.953 & $100 \%$ \\
\hline
\end{tabular}

Finally we consider the $2 \times 2 \times 2$-cube.

By inspecting fig. 4 we see that there are nine dimer coverings, to be divided into two classes, with weights $\tilde{c}_{1}$ and $\tilde{c}_{2}$.

In table IV we have summarized the results of calculations for this system. The first row contains the values for $\tilde{c}_{1}=\tilde{c}_{2}$. The second row gives the results obtained by minimizing the energy, the minimum corresponds with

$$
\left(\tilde{c}_{1} / \tilde{c}_{2}\right)_{\min }=\frac{2+\sqrt{13}}{3} .
$$

The exact ground-state energy for this system is ${ }^{19}$ )

$$
\varepsilon=-0.603 \text {. }
$$

Even in our crudest approximation we find a difference of only $2 \%$.
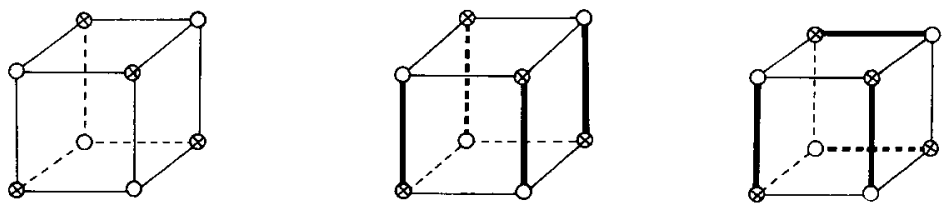

Fig. 4. The $2 \times 2 \times 2$-cube and its two types of dimer coverings. 


\section{Conclusions, expectations for large systems, discussion}

All small-system calculations learned us that the energy and the correlations belonging to the approximated ground state are not very sensitive to varying the weights of the dimer coverings in (2). This may be an indication that resonance is an important effect in our approximation. We believe that it is already a good approximation if we take equal weights in (2). In that case we not only satisfy Marshall's criterion, but we also give some plausibility to the expectation that some Ising configurations have a relatively large amplitude in the ground state.

From the results of the $4 \times 4$ square (especially those for periodic boundary conditions) we get the impression, by comparing our results with those of other authors, that our method provides us with reasonable estimates of some ground state properties (e.g. the energy).

To investigate the validity of our approximation for large systems we need other techniques, like Monte Carlo calculations, but some tendencies can already be noticed in small systems, and it is not difficult to see that they must be features of larger systems too.

In this context it is important to remember that there is a strong relation between the number of atoms and the dimension of the system at one side and the number of dimer coverings at the other side.

This has two consequences:

- When there are more coverings, the effect of resonance will be more important and we therefore expect to get a better approximation of the ground-state energy and the ground state itself.

- More coverings implicates a larger Néel amplitude, so we see, by using (14), (20) and (21), that in the case of high-dimensional systems, i.e. many dimer coverings, we may expect symmetry breaking to occur. In one dimension we are sure that there is no SB; in two dimensions it is possible that there is SB (unstable for $T>0$ ). Infinite-dimensional systems certainly have a ground state with SB.

Our conclusion is that our method may work well in large systems with a dimension $d \geqq 2$, but we need sophisticated calculations to decide whether it predicts long-range order (e.g. in the case $d=2$ ) or not. The accuracy of the method is expected to be increasing with increasing co-ordination number (dimension).

Resumé. We expect that this model, in which the ground state is approximated by a linear combination of singlet-pairs states only, for dimensions $d \geqq 2$ can give us insight in the phenomenon of long-range order as a geometric/ 
combinatorial effect. Also it is supposed to give a good estimate of the ground-state energy.

The authors have the opinion that this scheme can also be used to describe elementary excitations in Heisenberg systems, by introducing one triplet pair, in every dimer covering. States constructed along these lines have total spin $S=1$ and are orthogonal to the ground state.

\section{References}

1) H. Bethe, Z. Physik 71 (1931) 205.

2) E. Lieb, T. Schultz and D. Mattis, Ann. Phys. (N.Y.) 16 (1961) 407.

3) N.D. Mermin and H. Wagner, Phys. Rev. Lett. 17 (1966) 1133.

4) W. Marshall, Proc. Roy. Soc. (London) A232 (1955) 48.

5) H.L. Davis, Phys. Rev. 120 (1960) 789.

6) D.L. Bullock, Phys. Rev. A 137 (1965) 1877.

7) R. Kubo, Phys. Rev. A 87 (1952) 568.

8) R. Kubo, Rev. Mod. Phys. 25 (1953) 344.

9) R.R. Bartkowski, Phys. Rev. B 5 (1972) 4536.

10) J. Oitmaa and D.D. Betts, Can. Journ. Phys. 56 (1978) 897.

11) L.M. Floria and R. Navarro, J. Phys. C, Solid State Phys. 18 (1985) 6247.

12) W.J. van den Broek, Thesis, Twente University of Technology, Enschede (1985).

13) G.E. Hite, A. Metropoulos, D.J. Klein and T.G. Schmalz, preprint.

14) A.R. Edmonds, Angular Momentum in Quantum Mechanics (Princeton Univ. Press, Princeton, 1957), sec. 3.5 .

15) P.W. Anderson, Concepts in Solids (Benjamin, New York, 1963), sec. 3.D.4.

16) L. Hulthén, Arkiv Mat. Astron. Physik 26A (1938) 11.

17) P.W. Kasteleyn, J. Math. Phys. 4 (1963) 287.

18) M.E. Fisher, Phys. Rev. 124 (1961) 1664.

19) W.J. van den Broek, private communication, Twente University of Technology, Enschede (1982). 